\title{
Selective haploinsufficiency of longer isoforms of PTCH1 protein can cause nevoid basal cell carcinoma syndrome
}

\author{
Maiko Suzuki ${ }^{1}$, Hiromi Hatsuse ${ }^{1}$, Kazuaki Nagao ${ }^{1}$, Yoshinaga Takayama ${ }^{1}$, Kohzoh Kameyama ${ }^{1}$, \\ Yuji Kabasawa ${ }^{2}$, Ken Omura ${ }^{2}$, Masayuki Yoshida ${ }^{3}$, Katsunori Fujii ${ }^{4}$ and Toshiyuki Miyashita ${ }^{1}$
}

Nevoid basal cell carcinoma syndrome (NBCCS) is an autosomal dominant disorder characterized by developmental defects and tumorigenesis. The gene responsible for NBCCS is PTCH1. The PTCH1 gene has five alternatively used first exons resulting in the translation of three isoforms of the PTCH1 protein; that is, PTCHL, PTCHM and PTCHS. However, the biological significance of each isoform is unclear. Here we show an individual with NBCCS carrying a nonsense mutation in PTCH1 exon2, c.387G $>$ A (p.W129X). As the mutation lay upstream of the ATG codon used for PTCHS translation, the mutant allele still expressed RNA isoforms that encode PTCHS. These results clearly demonstrate that a selective haploinsufficiency of longer isoforms of the PTCH1 protein, PTCHL and PTCHM, but not PTCHS is sufficient to cause NBCCS. Although mice selectively deficient in PTCHS isoforms are currently unavailable, this study sheds light on the complex in vivo roles of PTCH1 isoforms. Journal of Human Genetics (2012) 57, 422-426; doi:10.1038/jhg.2012.45; published online 10 May 2012

Keywords: alternative splicing; nevoid basal cell carcinoma syndrome; PTCH1

\section{INTRODUCTION}

Nevoid basal cell carcinoma syndrome (NBCCS; OMIM 109400), also known as Gorlin syndrome, is an autosomal dominant disorder characterized by developmental defects including bifid ribs; palmar or plantar pits; and tumorigenesis such as the development of basal cell carcinoma, medulloblastoma or keratocystic odontogenic tumor (formerly known as odontogenic keratocysts)., ${ }^{1,2}$ The gene responsible for NBCCS is the human homolog of the Drosophilapatched gene, $P T C H 10^{3,4}$ The human $P T C H 1$ gene contains 23 coding exons spanning $\sim 65 \mathrm{~kb}$ and is predicted to encode a protein of 1447 amino-acid residues containing 12 transmembrane-spanning domains and 2 large extracellular loops. ${ }^{3}$

PTCH1, a 12-pass transmembrane protein, is the ligand-binding component of the hedgehog receptor complex. In the absence of hedgehog binding, PTCH1 is thought to hold Smoothened, a 7-pass transmembrane protein, in an inactive state and thus inhibit signaling to downstream genes. Upon the binding of hedgehog, the inhibition of Smoothened is released and signaling is transduced leading to the activation of target genes by the Gli family of transcription factors. ${ }^{5}$ The transcription of PTCH1 itself is induced by the hedgehog pathway activity, ${ }^{6}$ thus generating a negative feedback loop, which may have an important role in tumor suppression by inhibiting a sustained activation of the pathway.

Previously, we identified PTCH1 as one of the several genes undergoing markedly complicated alternative splicing. ${ }^{7}$ Focusing on the $5^{\prime}$ region of the PTCH1 gene, we found that alternative splicing takes place between five alternatively used first exons and one commonly used second exon (Figure 1a). ${ }^{8-10}$ This alternative splicing leads to the production of at least three isoforms of the PTCH1 protein, PTCHL, PTCHM and PTCHS (Figure 1b, wild-type allele). This alternative splicing is conserved in humans and mice suggesting a mechanism whereby a single $P T C H 1$ gene has a role in both tumor cell growth and embryonic development. However, the difference in the biochemical functions of these isoforms is still unclear. We demonstrated that PTCHS is more unstable than the other two probably because of degradation via the proteasome (unpublished data) ${ }^{8}$ As transgenic mice deficient in selected isoform(s) are currently unavailable, much less is known about the function of each isoform in vivo than that in vitro.

Here we show an individual with NBCCS carrying a nonsense mutation of PTCH1 exon 2 leading to a selective loss of PTCHL and PTCHM and discuss the roles of PTCH1 isoforms in vivo.

${ }^{1}$ Department of Molecular Genetics, Kitasato University Graduate School of Medical Sciences, Sagamihara, Japan; ${ }^{2}$ Department of Oral and Maxillofacial surgery, Graduate School, Tokyo Medical and Dental University, Tokyo, Japan; ${ }^{3}$ Life Science and Bioethics Research Center, Tokyo Medical and Dental University, Tokyo, Japan and ${ }^{4}$ Department of Pediatrics, Chiba University Graduate School of Medicine, Chiba, Japan

Correspondence: Dr T Miyashita, Department of Molecular Genetics, Kitasato University School of Medicine, 1-15-1 Kitasato, Minami-ku, Sagamihara 252-0374, Japan. E-mail: tmiyashi@med.kitasato-u.ac.jp

Received 31 August 2011; revised 26 March 2012; accepted 9 April 2012; published online 10 May 2012 
a

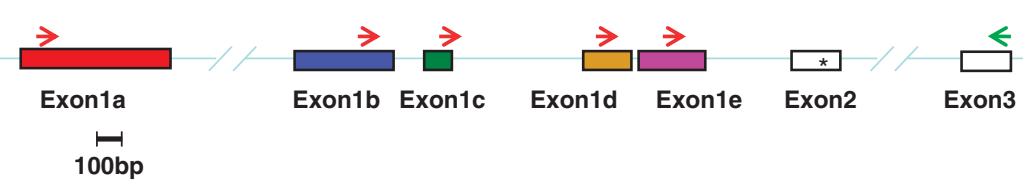

b

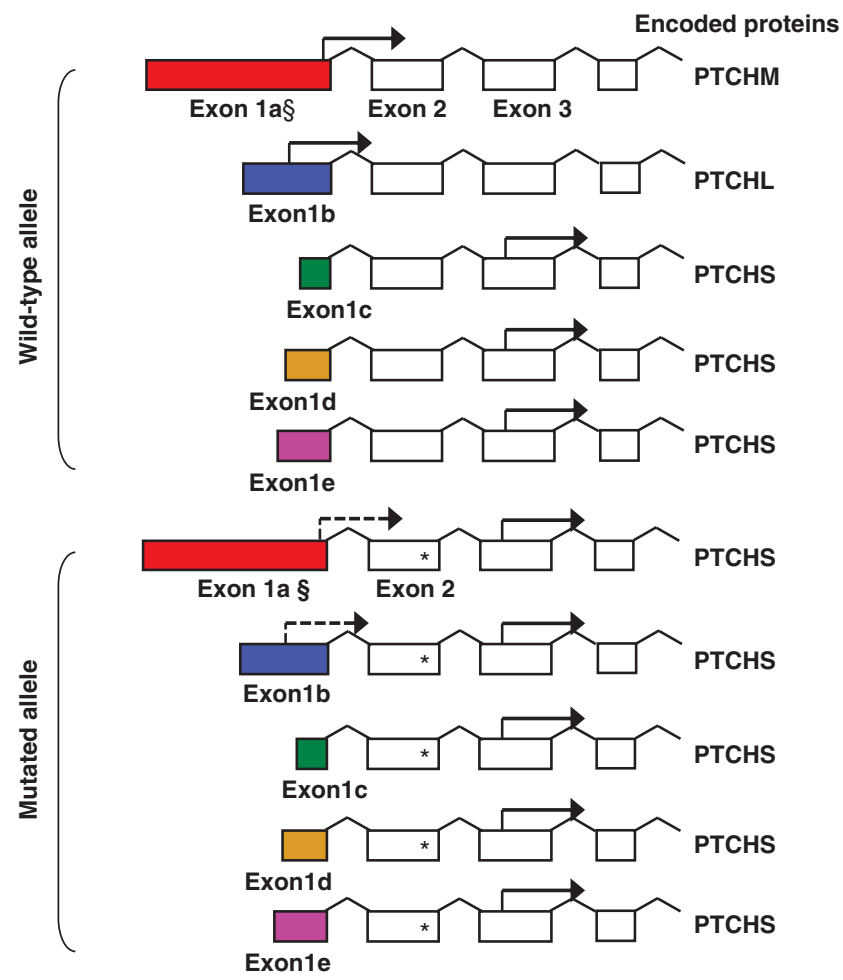

C

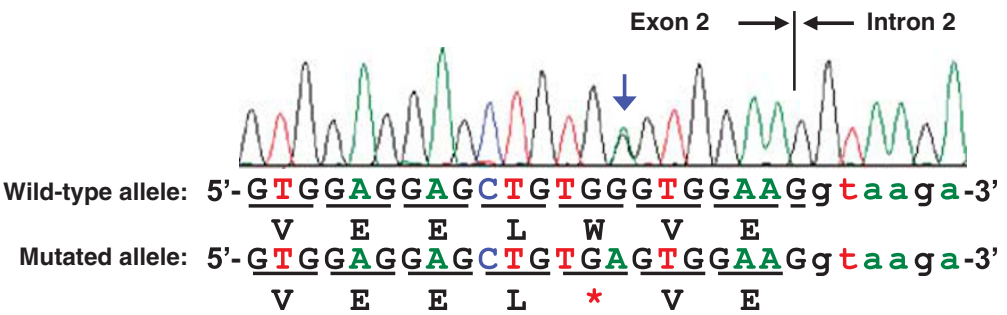

Figure 1 PTCH1 structure and mutation in exon2. (a) Genomic structure of $5^{\prime}$ region of the PTCH1 gene. Forward and reverse primers used for reversetranscription-PCR are indicated by red and green arrows, respectively. Mutation detected in this study is indicated by an asterisk. (b) Alternative splicing between the first and second exons. Translation initiation sites are represented by arrows. Translations of PTCHL and PTCHM start at exon 1b and exon 1a, respectively, whereas the translation of PTCHS starts at exon 3. Owing to the nonsense mutation, translations of PTCHL and PTCHM are terminated at PTC (dotted arrows). §: mRNA using exon 1 a as a first exon was not expressed in lymphoblastoid cell line. (c) Nonsense mutation detected in the patient.

\section{MATERIALS AND METHODS}

\section{Patient}

The patient, a 12-year-old boy, was an only child of healthy parents. At the time of diagnosis, he had three out of six major Kimonis criteria; ${ }^{11}$ that is, palmar and plantar pits, falx calcification and keratocystic odontogenic tumor. He also exhibited minor criteria such as macrocephaly and scoliosis. None of the phenotypes atypical for NBCCS was noted. He was, therefore, clinically diagnosed with NBCCS. No other family member had NBCCS phenotypes.

\section{Mutational analysis}

All experiments described below were approved by the ethics committee at Kitasato University. After written informed consent was obtained, blood samples were taken from the patient. DNA was extracted by using a QIAamp DNA blood midi kit (QIAGEN, Hilden, Germany). Genomic DNA samples were amplified with primers for all exons as described previously. ${ }^{12}$ Amplified products were gel-purified using a QIAEX II gel extraction kit (QIAGEN) and cycle-sequenced with a BigDye Terminator v3.1 Cycle Sequencing Kit (Applied Biosystems, Carlsbad, CA, USA) in both directions. The sequence was analyzed on a 3130 Genetic Analyzer (Applied Biosystems).

\section{Reverse-transcription-PCR analyses}

For the analysis of PTCH1 messenger RNA (mRNA) expression, an immortalized cell line was established by infection with Epstein-Barr virus obtained from B95-8 cells. Total RNA was extracted from an Epstein-Barr virusimmortalized lymphoblastoid cell line grown in the presence or absence of a translation inhibitor, puromycin $\left(100 \mu \mathrm{g} \mathrm{ml}^{-1}\right.$; Wako Pure Chemical, Osaka, Japan), for 6h, with a QIAamp RNA Blood Mini Kit (QIAGEN) and subjected to reverse-transcription-PCR using SuperScript III reverse transcriptase (Invitrogen, Carlsbad, CA, USA) with a random hexamer. The forward and 
reverse primers for exons $1 \mathrm{a}, 1 \mathrm{~b}, 1 \mathrm{c}, 1 \mathrm{~d}, 1 \mathrm{e}$ and 3 were $5^{\prime}$-AGCGCCTGTTTAC CCAGGAG-3', 5'-GGACCGGGACTATCTGCACC-3' ${ }^{\prime}, 5^{\prime}$-CCTCTCCAGGAAA AGCAGCA-3' ${ }^{\prime}, 5^{\prime}$-AAATGCCGCGCCGGGGAGCAGCCT-3' ${ }^{\prime} 5^{\prime}$-TTCTCGGCG GGGGTCCAGTT- $3^{\prime}, 5^{\prime}$-GTATACATGGACACGGCTGGCC- $3^{\prime}$ and $5^{\prime}$-AGCCT CTTCTCCAATCTTCTGG- $3^{\prime}$, respectively (Figure 1a). The amplified products were subcloned into the pGEM-T Easy vector (Promega, Madison, WI, USA) and sequenced.

\section{Plasmid construction}

The expression construct encoding the $\mathrm{PTCH} 1$-enhanced green fluorecence protein (EGFP) fusion proteins, pPTCHM-EGFP and pPTCHS-EGFP were described previously. ${ }^{13}$ pPTCHW129X-EGFP encoding a mutant version of PTCHM-EGFP was generated by PCR-mediated mutagenesis using a pair of primers, 5'-GTGGAAGTTGGAGGACGAGT- $3^{\prime}$ and $5^{\prime}$-TCACAGCTCCTCCAC GTTGG-3' (mutated base is underlined).

\section{Transfection and immunoblotting}

The human embryonic cell line 293 was transfected with pPTCHM-EGFP, pPTCHS-EGFP or pPTCHW129X-EGFP, using Effectene transfection reagent (QIAGEN). Sixteen hours after the transfection, the cells were further cultured in the presence or absence of a proteasome inhibitor, MG132 $(10 \mu \mathrm{M}$; Peptide Institute, Osaka, Japan) for $6 \mathrm{~h}$. Thirty micrograms of cell lysate was subjected to SDS-polyacrylamide gel electrophoresis and immunoblotting using rabbit anti-GFP antibody (Medical \& Biological Laboratories, Nagoya, Japan), followed by horseradish peroxidase-conjugated anti-rabbit IgG (Santa Cruz, Santa Cruz, CA, USA). Expressed proteins were visualized with an enhanced chemiluminescence (ECL) Western Blotting Detection System (GE Healthcare, Buckinghamshire, UK) using an LAS-4000 luminescent image analyzer (GE Healthcare). The amounts of expressed proteins were quantified by ImageQuant TL software (GE Healthcare).

\section{RESULTS}

\section{PTCH1 mutation}

A heterozygous PTCH1 mutation, c. $387 \mathrm{G}>\mathrm{A}$, was detected in this patient (Figure 1c). It caused a nonsense mutation resulting in the premature termination of the PTCH1 protein (p.W129X). The parents did not carry this mutation. Thus, the mutation was concluded to be generated de novo. As it lies in exon 2, PTCHL and PTCHM cannot be expressed from the mutant allele because of the premature termination codon. In contrast, translation of PTCHS starts at an initiation codon located in exon 3, which is downstream of the mutation. Therefore, PTCHS can still be expressed from the mutant allele theoretically (Figure 1b).

\section{Expression of the mutant RNA}

To explore the abovementioned possibility, we performed reversetranscription-PCR using one of the forward primers designed for each 1 st exon and a reverse primer designed for exon 3 (Figure 1a). mRNA species starting from exon $1 \mathrm{~b}, 1 \mathrm{c}, 1 \mathrm{~d}$ and $1 \mathrm{e}$ were amplified from total RNA extracted from a lymphoblastoid cell line established from the patient. Exon 1a was not expressed in these cells. This is consistent with our previous observation that the expression of exon la was rather restricted. ${ }^{8}$ Furthermore, the absence of exon 1a expression was not specific to this patient, because lymphoblastoid cell lines established from other individuals did not express exon 1a either. We then subcloned the reverse-transcription-PCR product into the pGEM-T Easy vector and sequenced the insert to distinguish wildtype and mutant mRNAs.

mRNA species harboring premature termination codons undergo a form of degradation called nonsense-mediated mRNA decay (NMD).${ }^{14}$ NMD is a highly conserved surveillance process leading to the detection and selective reduction of premature termination codon-harboring mRNAs to prevent the synthesis of abnormal
Table 1 Wild-type (Wt) and mutant (Mt) mRNA sequences detected by reverse-transcription-PCR

\begin{tabular}{ccccc}
\hline Sequence & Wt & Mt & Wt & Mt \\
\hline Puromycin & - & - & + & + \\
Splicing & & & & \\
1a-2-3 & ND & ND & ND & ND \\
1b-2-3 & 7 & 11 & 8 & 10 \\
1c-2-3 & 18 & 9 & 13 & 14 \\
1d-2-3 & 10 & 10 & 10 & 12 \\
1e-2-3 & 7 & 9 & 11 & 7
\end{tabular}

Reverse-transcription-PCR products were subcloned into the pGEM-T Easy vector and Escherichia coli was transformed. DNA was extracted from the transformed $E$. coli and sequenced. The number of colonies detected in each category is indicated.

proteins. As NMD is a translation-dependent process, the degradation is suppressed by translation inhibitors such as puromycin. ${ }^{15}$

Regardless of the usage of the first exon, the mutant sequence as well as the wild-type was detected in subcloned constructs (Table 1). The addition of puromycin to the culture medium did not influence the ratio of wild-type to mutant sequences. These results demonstrate that the mutant RNA isoforms are NMD-insensitive. This is not surprising for RNA species using exon $1 \mathrm{c}$, $1 \mathrm{~d}$ or $1 \mathrm{e}$ because the mutation is located upstream of the initiation codon and, therefore, is not regarded as a premature termination codon. In contrast, in the isoform using exon $1 \mathrm{~b}$, the initiation codon is in the first exon and the mutation lies downstream. Therefore, it was unexpected that this isoform was also NMD-insensitive. This prompted us to test whether the translation starts at the second ATG codon in exon 3 in the mutant allele.

\section{Protein translation from the mutant allele}

To investigate the abovementioned possibility, 293 cells were transfected with any of the plasmids encoding PTCHM-EGFP, PTCHSEGFP and a mutant version of PTCHM-EGFP (pPTCHW129XEGFP) carrying a nonsense mutation downstream of the first ATG codon (Figure 2a). When the wild-type PTCHM-EGFP plasmid was introduced into 293 cells, the fusion protein was overexpressed in these cells as described previously (Figure $2 \mathrm{~b}$, lane 1). ${ }^{13}$ A fusion protein with a lower molecular weight corresponding to that of PTCHS-EGFP was expressed when pPTCHW129X-EGFP was introduced (Figure 2b, lanes 3 and 5). As we reported previously, the expression level of PTCHS-EGFP was lower than that of PTCHMEGFP owing to the reduced stability of the PTCHS protein. ${ }^{8}$ We next treated transfected cells with a proteasome inhibitor, MG132. MG132 markedly increased the expression levels of fusion proteins translated from pPTCHS-EGFP and pPTCHW129X-EGFP, but not pPTCHMEGFP (Figure 2b, lanes 1, 2, 5 and 6). Taken together, the mutation, c.387G > A (p.W129X), resulted in translation from the second initiation codon generating a PTCHS instead of PTCHM isoform, the former undergoing degradation by the proteasome.

\section{DISCUSSION}

The $5^{\prime}$ structure of the human PTCH1 gene, had been unclear until we and others elucidated that there exist 5 exons alternatively used as a first exon, resulting in the production of at least 3 protein isoforms. ${ }^{8-10}$ As the $5^{\prime}$ exonic structure of PTCH1 is conserved in humans and mice, alternative splicing between the first and second 
a

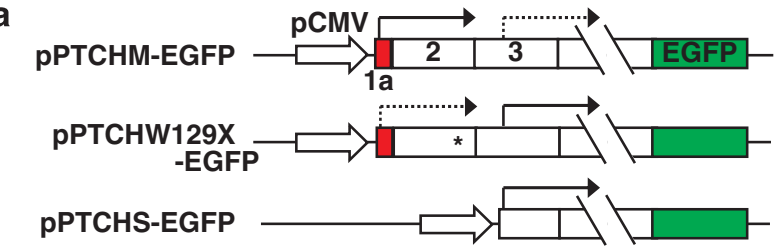

b

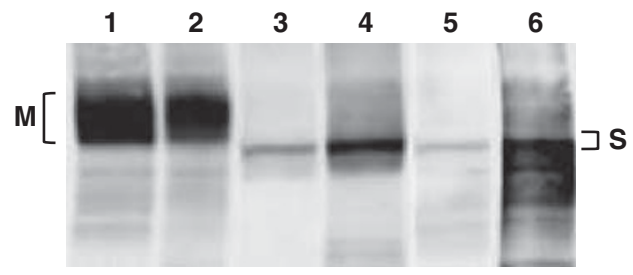

C
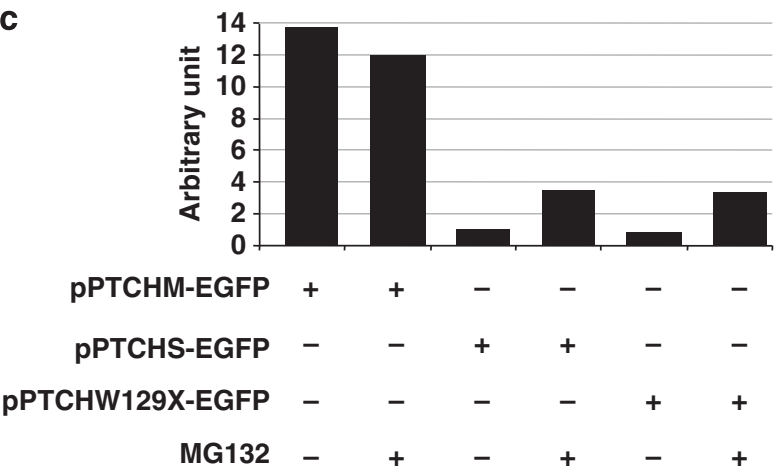

Figure 2 Immunoblotting of fusion proteins. (a) Schematic representation of the expression constructs. pCMV, cytomegalovirus promoter (b) 293 cells were transfected with either pPTCHM-EGFP, pPTCHS-EGFP or pPTCHW129X-EGFP. After the transfection, cells were grown with or without a proteasome inhibitor, MG132, for $6 \mathrm{~h}$. Expressed fusion proteins were detected with anti-GFP antibody. (c) Quantification of expressed proteins. Expressed proteins were quantified by ImageQuant TL software.

exons seems to have an important role in embryonic development and carcinogenesis. Indeed, the usage of these exons is differentially regulated, both temporally and spatially. ${ }^{8}$ We also reported that the short isoform of the PTCH1 protein (PTCHS) is less stable than the others. ${ }^{8}$ However, the difference in biological roles of these isoforms has been unclear.

Here, we report a patient carrying a nonsense mutation in exon 2. This mutation is located downstream of the initiation codon used for the translation of PTCHL and PTCHM, but upstream of that used for PTCHS production. As far as we know, at least 3 cases of NBCCS harboring nonsense mutations in exon 2 have been reported so far. ${ }^{16,17}$ However, a detailed study of the expression of each isoform in these cases has never been performed.

First of all, PTCHS is expected to be produced from mutant mRNA species in which exon 1c, 1d or 1e is used as the first exon (Figure 1b). Although we do not have experimental evidence, it is likely that the amount of PTCHS produced from these mutant mRNAs is similar to that produced from the wild-type RNA because the difference in the sequence is only one base substitution at $67 \mathrm{bp}$ upstream of the ATG codon for PTCHS. Furthermore, no difference in RNA secondary structure prediction was observed when analyzed by GENETYX software (GENETYX Corporation, Tokyo, Japan; data not shown).

Therefore, we focused on the translational events in the wild-type and mutant mRNAs in which exon $1 \mathrm{a}$ is used as the first exon corresponding to pPTCHM-EGFP and pPTCHW129X-EGFP, respectively (owing to the GC-rich sequence, we were unable to produce the expression construct in which exon $1 \mathrm{~b}$ is used as the first exon). When pPTCHM-EGFP construct was introduced, PTCHMEGFP fusion protein was produced (Figure $2 b$, lane 1 ). However, the fusion protein corresponding to PTCHS-EGFP was not observed, at least not in our experimental system (compare Figure 2b, lane 1 with 3). Therefore, first ATG codon is suggested to be used almost exclusively in this situation. In contrast, as expected, no PTCHM fusion protein was translated from a mutant version of pPTCHMEGFP (pPTCHW129X-EGFP) because of the termination codon created by the nonsense mutation, p.W129X (Figure 2b, lane 5). Instead, although the expression level was low, PTCHS-EGFP was produced from this construct by using the second ATG codon downstream of the mutation. The usage of the second initiation codon was confirmed by the size of the fusion protein, which corresponded to PTCHS-EGFP (compare Figure 2b, lanes 3 with 5).

Taken together, the mutant cells in this patient would produce the half amount of longer isoforms (PTCHM and L) but the similar or even a bit higher amount of PTCHS. Thus, NBCCS is suggested to develop because of a haploinsufficiency of PTCHL and PTCHM but not PTCHS.

The role of PTCHS in vivo is still unclear. This case might tell us that PTCHS is dispensable. However, the mRNA species encoding PTCHS is more ubiquitously expressed than that of any other PTCH1 isoform in human tissues. ${ }^{8}$ Taking its instability into account, PTCHS may have a role in situations where the transient expression and rapid degradation of $\mathrm{PTCH} 1$ are required.

Genetically engineered mice deficient in Ptch1 have already been generated. ${ }^{18}$ Mice homozygous for the Ptch 1 mutation die during embryogenesis and mice heterozygous for the Ptch1 mutation are predisposed to cancers such as medulloblastoma serving as a model for NBCCS. However, mice deficient in certain isoforms of Ptch1 are currently unavailable. Therefore, this study provides a valuable clue as to the distinct roles of each PTCH1 isoform. Although no NBCCS patients harboring a mutation in one of the five $5^{\prime}$ exons have ever been identified, any such cases would shed more light on the distinct roles of $\mathrm{PTCH} 1$ isoforms in vivo.

\section{ACKNOWLEDGEMENTS}

We are grateful to the participating family members whose contributions made this study possible. This research was supported by Science Research Grants for intractable diseases in Japan (H22-intractable diseases-120) from the Ministry of Health, Labour and by a Grant-in-Aid for Science Research (20591261) from the Ministry of Education, Culture, Sports, Science and Technology.

1 Gorlin, R. J. \& Goltz, R. W. Multiple nevoid basal-cell epithelioma, jaw cysts and bifid rib. A syndrome. N. Engl. J. Med. 262, 908-912 (1960).

2 Gorlin, R. J. Nevoid basal-cell carcinoma syndrome. Medicine (Baltimore) 66, 98-113 (1987).

3 Johnson, R. L., Rothman, A. L., Xie, J., Goodrich, L. V., Bare, J. W., Bonifas, J. M. et al. Human homolog of patched, a candidate gene for the basal cell nevus syndrome. Science 272, 1668-1671 (1996).

4 Hahn, H., Wicking, C., Zaphiropoulous, P. G., Gailani, M. R., Shanley, S., Chidambaram, A. et al. Mutations of the human homolog of Drosophila patched in the nevoid basal cell carcinoma syndrome. Cell 85, 841-851 (1996).

5 Ingham, P. W. \& McMahon, A. P. Hedgehog signaling in animal development: paradigms and principles. Genes Dev. 15, 3059-3087 (2001).

6 Berman, D. M., Karhadkar, S. S., Hallahan, A. R., Pritchard, J. I., Eberhart, C. G., Watkins, D. N. et al. Medulloblastoma growth inhibition by hedgehog pathway blockade. Science 297, 1559-1561 (2002).

7 Nagao, K., Togawa, N., Fujii, K., Uchikawa, H., Kohno, Y., Yamada, M. et al. Detecting tissue-specific alternative splicing and disease-associated aberrant splicing of the PTCH gene with exon junction microarrays. Hum. Mol. Genet. 14, 3379-3388 (2005). 
8 Nagao, K., Toyoda, M., Takeuchi-Inoue, K., Fujii, K., Yamada, M. \& Miyashita, T. Identification and characterization of multiple isoforms of a murine and human tumor suppressor, patched, having distinct first exons. Genomics 85, 462-471 (2005).

9 Kogerman, P., Krause, D., Rahnama, F., Kogerman, L., Undén, A. B., Zaphiropoulos, P. G. et al. Alternative first exons of PTCH1 are differentially regulated in vivo and may confer different functions to the PTCH1 protein. Oncogene 21, 6007-6016 (2002).

10 Shimokawa, T., Rahnama, F. \& Zaphiropoulos, P. G. A novel first exon of the Patched 1 gene is upregulated by Hedgehog signaling resulting in a protein with pathway inhibitory functions. FEBS Lett. 578, 157-162 (2004).

11 Kimonis, V. E., Goldstein, A. M., Pastakia, B., Yang, M. L., Kase, R., DiGiovanna, J. J. et al. Clinical manifestations in 105 persons with nevoid basal cell carcinoma syndrome. Am. J. Med. Genet. 69, 299-308 (1997).

12 Fujii, K., Kohno, Y., Sugita, K., Nakamura, M., Moroi, Y., Urabe, K. et al. Mutations in the human homologue of Drosophila patched in Japanese nevoid basal cell carcinoma syndrome patients. Hum. Mutat. 21, 451-452 (2003).

13 Uchikawa, H., Toyoda, M., Nagao, K., Miyauchi, H., Nishikawa, R., Fujii, K. et al. Brain- and heart-specific Patched-1 containing exon $12 \mathrm{~b}$ is a dominant negative isoform and is expressed in medulloblastomas.. Biochem. Biophys. Res. Commun. 349, 277-283 (2006).

14 Holbrook, J. A., Neu-Yilik, G., Hentze, M. W. \& Kulozik, A. E. Nonsense-mediated decay approaches the clinic. Nat. Genet. 36, 801-808 (2004).

15 Carter, M. S., Doskow, J., Morris, P., Li, S., Nhim, R. P., Sandstedt, S. et al. A regulatory mechanism that detects premature nonsense codons in T-cell receptor transcripts in vivo is reversed by protein synthesis inhibitors in vitro. J. Biol. Chem. 270, 28995-29003 (1995).

16 Savino, M., d'Apolito, M., Formica, V., Baorda, F., Mari, F., Renieri, A. et al. Spectrum of PTCH mutations in Italian nevoid basal cell-carcinoma syndrome patients: identification of thirteen novel alleles. Hum. Mutat. 24, 441 (2004).

17 Minami, M., Urano, Y., Ishigami, T., Tsuda, H., Kusaka, J. \& Arase, S. Germline mutations of the PTCH gene in Japanese patients with nevoid basal cell carcinoma syndrome. J. Dermatol. Sci. 27, 21-26 (2001).

18 Goodrich, L. V., Milenković, L., Higgins, K. M. \& Scott, M. P. Altered neural cell fates and medulloblastoma in mouse patched mutants. Science 277, 1109-1113 (1997). 